\title{
Les changements politiques et psychologiques suscités par la guerre de Corée Holly Bray
}

L'année était 1950. Le rideau de fer, expression devenue célèbre par le premier ministre britannique Churchill, était déjà dressé. La Guerre froide venait de commencer, et la machinerie politique mondiale grondait sous les conséquences accablantes d'une guerre mondiale et la tension entre deux de ses vainqueurs : 1'Union soviétique et les États-Unis. Ces derniers, luttant férocement pour la propagation de leurs propres idées politiques et économiques à travers le monde, contrôlaient chacun une moitié de la Corée avec leurs chefs politiques alliés soigneusement mis en place. Mais cet équilibre a été brisé le 25 juin 1950, quand les forces armées nord-coréennes ont franchi le trente-huitième parallèle pour tenter l'unification du pays du Matin calme sous un régime communiste. Ce premier affrontement a marqué le commencement d'un conflit sanglant en Corée, qui n'a pas terminé qu'après des centaines de sessions de négociation et trois années de combats. Quoique l'issue de la guerre de Corée était quasiment un retour au statut ante bellum, elle a eu des répercussions profondes sur la Guerre froide et sur les stratégies militaires et politiques des États-Unis dans celle-ci.

Ma dissertation étudiera comment la guerre de Corée a transformé le climat de la Guerre froide pour les États-Unis. Même si ce conflit n'était qu'un de plusieurs affrontements qui ont éclaté pendant la Guerre froide, on ne peut pas négliger son empreinte sur les agissements politiques de cette époque. En effet, cette guerre a beaucoup contribué aux tensions déjà existantes entre le bloc communiste et le bloc occidental malgré son résultat, en apparence seulement, nul pour les deux côtés. L'absence de l'émergence d'un vainqueur dans la guerre de Corée évoque la complexité de cette dernière et nous montre que ce conflit de trois ans mérite notre attention.

Je consacrerai la première partie de ma dissertation à une analyse des changements politiques provoqués par la guerre de Corée, puisqu'il est nécessaire de comprendre les relations politiques qui existaient entre les adversaires et leurs alliés pour voir comment cette guerre se différencie de celle qui l'a précédée cinq années auparavant. Celle-ci sera suivie d'une partie qui décrira les changements psychologiques pour les Américains face aux difficultés que la guerre de Corée leur a présentées, notamment la façon dont leurs stratégies militaires ont été mises en question.

Concentrons-nous d'abord sur l'aspect politique de la guerre de Corée. L'historien américain Gabriel Kolko a décrit qu'à cette époque aux États-Unis, il se passait « une transformation pour ainsi dire exponentielle de l'art de la guerre ${ }^{1}$, et c'était cette transformation qui a façonné la conduite des États-Unis dans la guerre de Corée et dans la Guerre froide. Après la fin de la Deuxième Guerre mondiale, l'harmonie au sein des Alliés n'existait plus suite au schisme qui s'est produit avec l'Union soviétique. Cependant, ses membres ont rapidement remplacé cette alliance de guerre et l'inefficace Société des Nations par une nouvelle société politique internationale, l'Organisation des Nations unies (ONU). En réponse à Deux Guerres mondiales dévastatrices, ses priorités étaient la diplomatie et la coopération internationale afin de prévenir un autre conflit à cette échelle. Mais ce qui a changé la politique américaine sur le plan de la guerre était la question de l'aide mutuelle et la solidarité militaire des États membres de l'ONU. Dans le cas d'un conflit, des soldats de plusieurs pays pourraient se joindre sous le drapeau onusien contre l'agresseur. Cette clause a accordé bien sûr la protection militaire aux pays moins

\footnotetext{
${ }^{1}$ Gabriel Kolko, Un siècle de guerres : Politique, conflit et société depuis 1914 (Saint-Nicolas, QC : Les presses de l'Université Laval, 1994), 343.
} 
développés avec de forces armées minimes, mais elle a aussi modifié la façon dont un pays puissant comme les États-Unis sont entrés en guerre en 1950. L'unité militaire des Nations unies a changé la dynamique politique des Américains; ce nouveau système a inspiré beaucoup (et peut-être trop) de confiance au sein de la branche exécutive, notamment chez le président américain Harry S. Truman. Dans un discours prononcé le $1^{\text {er }}$ septembre 1950 au peuple américain, le président Truman a évoqué ce sentiment de confiance dans la puissance de l'ONU quand il a déclaré que l'impérialisme communiste « can be discouraged from new aggression if we and other free peoples are strong, determined and united $»^{2}$ et, plus explicitement, "We believe in the United Nations. [...] [W] pledged ourselves to seek peace and security through this world organisation. [...] We shall never go back on that pledge. ${ }^{3}$ D'ailleurs, il faut mentionner que le système de vote aux Nations unies a été manipulé par les Américains à deux reprises pour obtenir le support diplomatique nécessaire d'aller en Corée ; ils ont profité d'une absence du délégué soviétique pour condamner l'agression nord-coréenne, et ils ont choisi de renvoyer le vote sur l'action militaire en Corée à l'Assemblée générale, au lieu de voter au Conseil de sécurité où l'Union soviétique aurait pu utiliser son véto. ${ }^{4}$ On doit aussi se souvenir que les États-Unis sont sortis parmi les vainqueurs pour les deux Guerres mondiales, et que ces expériences de succès à l'échelle internationale ont largement contribué à une surestimation de soi parmi les politiciens américains. La croyance des États-Unis à l'omnipotence d'une armée internationale (ou de l'armée américaine) a été toutefois dissipée lors de la prolongation de la guerre de Corée. Si, par chance, les États-Unis ont oublié l'astuce d'une Union soviétique qui a écrasé l'armée allemande en 1945, ils se sont souvenus après quelques mois en Corée. Malgré le fait que la force économique résidait dans le bloc occidental, les forces armées nord-coréennes avaient l'armement soviétique et elles avaient été sous la tutelle de l'armée soviétique pour plusieurs années pendant l'occupation militaire du territoire. De plus, l'Union soviétique venait de gagner un allié en 1949, la Chine communiste, qui pouvait lui offrir le soutien militaire et politique. Elle aussi a été sous-estimée par les États-Unis, manque d'expérience avec l'armée chinoise formidable. Même si Truman désirait éviter une guerre avec la Chine, l'industrialisation retardataire, l'armement insuffisant et l'instabilité politique d'une Chine récemment bouleversée par une révolution a fait que la menace n'était pas prise au sérieux. ${ }^{5}$ La participation des Chinois dans la guerre de Corée a cependant détruit cette illusion, après que la majorité des avances territoriales de l'ONU ont été perdues avec l'offensif chinois. Par conséquent, l'influence des Chinois et des Soviétiques en Corée a provoqué un changement politique pour les États-Unis puisque l'idée de l'omnipotence du bloc occidental s'est avérée chimérique dans le contexte de la guerre. Le troisième changement s'est réalisé suite à l'utilisation des pions politiques des deux côtés. Certainement la notion d'un pion politique n'était pas une notion innovante ni exclusive à la guerre de Corée ; néanmoins, c'était l'implication d'autres acteurs sur la scène politique qui a empêché que la Guerre froide soit transformée en guerre directe. Examinons d'abord l'entrée en guerre des États-Unis par l'intermédiaire des Nations unies. Les facteurs qui ont influencé cette décision étaient tout à fait politiques, puisque c'étaient les États-Unis qui avaient la puissance économique du bloc occidental. On peut croire que la solidarité onusienne a été une protection pour que les États-Unis ne soient pas impliqués dans une guerre directe avec l'Union soviétique,

\footnotetext{
${ }^{2}$ Harry S. Truman, “Our Aims and Our Hopes," Vital Speeches of the Day 16, no. 3 (1950): 712.

${ }^{3}$ Ibid., 711.

${ }^{4}$ André Fontaine, La guerre froide : 1917-1991 (Paris : Éditions de la Martinière, 2006), 149.

${ }^{5}$ Alexander Ovodenko, "(Mis)interpreting Threats: A Case Study of the Korean War," Security Studies 16, no. 2 (2007): 279.
} 
ou que cette dernière craindrait un conflit avec toutes les nations réunies contre elle. D'ailleurs, le rôle d'un agresseur solitaire, venu du côté des Alliés, n'aurait pas été acceptable politiquement après six années de guerre mondiale, d'où la condamnation de l'agression nord-coréenne. Les évènements de juin $1950 \mathrm{a}$, au contraire, permis aux États-Unis de jouer le rôle d'une victime. Ceci était évident dans le discours de Truman en 1950, dans lequel il a expliqué que «the free nations have been compelled to take measures to protect themselves against the aggressive designs of the Communists. " ${ }^{6}$ De l'autre côté du rideau de fer, l'URSS a su manipuler ses alliés pour empêcher l'entrée en guerre directe, elle aussi. Contrairement aux analyses de Washington, la Chine communiste n'était pas soumise aux ordres de Moscou malgré le traité sino-soviétique d'aide mutuelle. ${ }^{7}$ Mais l'absence d'une réaction menaçante des Soviétiques après le premier offensif de l'ONU en Corée n'était pas une coïncidence. L'Union soviétique savait très bien que la situation ne méritait pas une agression claire de sa part, puisque la Chine était plus vulnérable géographiquement et agirait en défense légitime si nécessaire. ${ }^{8}$ Les Nations unies ne contemplaient pas la possibilité d'une guerre avec un pays si puissant que l'URSS, mais on discutait d'un bombardement de la Mandchourie, surtout avec l'Américain d'un instinct belliqueux, Douglas MacArthur, comme chef des forces armées. De cette façon, la Chine a réussi à défendre la Corée du Nord sans que l'Union soviétique soit impliquée directement. Le bloc occidental et le bloc communiste ont aussi mis en place des chefs coréens fidèles à l'idéologie politique de chaque superpuissance : Kim Il-Sung en Corée du Nord et Syngman Rhee en Corée du Sud. L'établissement d'un chef pro-occidental n'était rien de nouveau pour les Américains, mais leur relation avec ce chef coréen redoutable, surnommé « le Vieillard terrible », a beaucoup influencé la guerre et a présenté aux États-Unis un défi politique. D’après Gabriel Kolko, « [1]es deux Coréens les plus importants, [...] étaient suffisamment indépendants de ceux qui, à l'origine, semblaient être à la fois leurs bailleurs de fonds et leurs puissants maitres, pour fausser les calculs et faire fi des désirs de ces derniers» ${ }^{9}$, et cet énoncé s'est avéré exact avec le cas de Syngman Rhee et les forces de l'ONU. Quoique Rhee coopérait avec le bloc occidental, il était sans question que son but principal était de réunir les deux Corées à tout prix : il est allé même jusqu'à sous-miner les négociations de paix entre la Chine et les Nations unies. ${ }^{10}$ La réunification des deux Corées ne s'est jamais réalisée, mais Rhee a su comment convaincre les États-Unis et l'ONU à investir leur argent, leur armée et leur temps pour la tenter. Les États-Unis étaient habitués à faire la diplomatie avec des chefs qui savaient avec qui ils avaient affaire ; la situation avec Syngman Rhee a changé cela et les Américains ont dû s'adapter à un nouveau défi politique. Ces changements politiques qui ont été provoqués par la guerre de Corée ont tous eu un impact sur la Guerre froide et la façon de laquelle les États-Unis ont su s'adapter à des situations difficiles, agir dans la communauté internationale, et adopter un point de vue réaliste au sujet de leur propre puissance.

\footnotetext{
${ }^{6}$ Harry S. Truman, “Our Aims and Our Hopes,” Vital Speeches of the Day 16, no. 3 (1950): 710.

7 Alexander Ovodenko, “(Mis)interpreting Threats: A Case Study of the Korean War," Security Studies 16, no. 2 (2007): 274.

${ }^{8}$ Ibid., 275.

${ }^{9}$ Gabriel Kolko, Un siècle de guerres : Politique, conflit et société depuis 1914 (Saint-Nicolas, QC : Les presses de l'Université Laval, 1994), 346.

${ }^{10}$ André Fontaine, Histoire de la guerre froide : De la guerre de Corée à la crise des alliances 1950-1963 (Paris : Librairie Arthème Fayard, 1967), 79.
} 
La guerre de Corée a changé les États-Unis sur le plan politique, mais une analyse des changements suscités par ce conflit ne serait jamais complète sans une étude de l'aspect psychologique de cette guerre. Après la Deuxième Guerre mondiale, les Américains étaient dans une position de redresser l'Europe et de connaître un grand succès économique domestique. Pourtant, ce sentiment d'assurance et d'optimisme était supprimé par les révélations de la guerre de Corée et leur impact sur l'état psychologique des États-Unis. En premier lieu, cette guerre a éprouvé les capacités diplomatiques et la patience des Nations unies, puisqu'elle requérait beaucoup de discrétion politique pour éviter qu'elle soit une guerre totale. Cinq années après la fin d'un conflit mondial, c'était dans l'intérêt des États-Unis et des Nations unies d'empêcher que cela arriverait de nouveau. Dans le discours de Truman «Our Aims and Our Hopes », le président a déclaré explicitement qu'il ne voulait pas que la guerre en Corée se dégraderait en guerre totale, toutefois élaborant sur la nécessité d'action militaire. Des phrases comme " [i]f aggression were allowed to succeed in Korea, it would be an open invitation to new acts of aggression elsewhere ${ }^{11}$ et "our country has been called upon to give of its leadership, its efforts and its resources, to maintain peace and justice among nations $\gg{ }^{12}$ évoquent la doctrine Truman, selon laquelle le communisme devait être endigué et que les États-Unis avaient la responsabilité d'effectuer cet endiguement. Pourtant, la situation en Corée n'avait pas de buts si simples et clairs. Comme l'auteur Alexander Ovodenko a constaté, la guerre était en effet plus complexe : si les forces armées onusiennes réussiraient à regagner le territoire sud-coréen perdu pour revenir au statu quo ante bellum au trente-huitième parallèle, la menace d'une invasion nord-coréenne existerait toujours après que l'armée se retirerait. ${ }^{13}$ Et si on tenterait une réunification des deux Corées, cela provoquerait peut-être la colère de la Chine ou de l'URSS. L'ONU a été alors placée dans une situation extrêmement délicate, où il fallait juger le moment propice pour chaque mouvement avec soin. Ni les Nations unies ni les États-Unis ne pourraient souffrir les pertes économiques ou humaines d'une guerre avec l'Union soviétique, les Américains avant tout puisqu'une guerre prolongée en Corée ou dans la région du Pacifique laisserait l'Europe aux manœuvres de Staline. De plus, l'armement depuis la Deuxième Guerre mondiale a changé pour inclure les armes nucléaires, ce qui a donné à la Guerre froide un élément de risque encore plus extrême qu'une guerre totale sans la bombe atomique ou thermonucléaire. Les deux côtés étant équipés de cette technologie-ci (les Soviétiques essayaient leur bombe aussi tôt que 1949), un conflit direct résulterait en de graves conséquences pour les belligérants et pour l'humanité. La prudence diplomatique était alors nécessaire, jusqu'au point où une absence de celle-ci a mené à la destitution du général MacArthur, le chef commandant américain des forces armées des Nations unies. Les commentaires belliqueux, l'encouragement vif de recourir à l'arme nucléaire et la mésentente avec la branche exécutive américaine de MacArthur n'ont fait qu'empirer les conditions diplomatiques et caractériser le général célèbre comme étant un baril de poudre prêt à éclater. ${ }^{14}$ Le comportement de MacArthur, et la réaction de Washington à celui-ci, a illustré le changement psychologique entre l'attitude plus agressive employée autrefois et l'attitude diplomatique que la guerre de Corée a nécessité. Un autre facteur qui a modifié l'esprit américain face à la guerre était que l'industrialisation d'un pays n'apportait

\footnotetext{
${ }^{11}$ Harry S. Truman, “Our Aims and Our Hopes,” Vital Speeches of the Day 16, no. 3 (1950): 710.

12 Ibid., 712.

${ }^{13}$ Alexander Ovodenko, “(Mis)interpreting Threats: A Case Study of the Korean War," Security Studies 16, no. 2 (2007): 265.

${ }^{14}$ André Fontaine, Histoire de la guerre froide : De la guerre de Corée à la crise des alliances 1950-1963 (Paris : Librairie Arthème Fayard, 1967), 33.
} 
pas nécessairement la victoire sur le champ de bataille. Si la Deuxième Guerre mondiale a montré aux États-Unis le pouvoir d'une économie forte, la guerre de Corée leur a montré ses faiblesses. En 1950, Truman a constaté dans son discours que "Hitler and the Japanese miscalculated badly [...] when they thought we would not be able to use our economic power effectively to defeat aggression. Let would-be aggressors make no such mistake today. " ${ }^{15} \mathrm{C}$ 'était en effet la preuve qu'on croyait que les ressources économiques allaient acheter aux États-Unis la victoire, peu importe d'adversaire. Cette notion était une notion dangereuse, surtout dans le contexte du réarmement pendant la Guerre froide. Ce réarmement s'est organisé aux États-Unis selon le NSC-68: essentiellement un document passé par le Conseil de sécurité nationale américain pour augmenter le budget militaire jusqu'à vingt pourcent du budget fédéral et pour réarmer les États-Unis pour qu'ils pourraient faire face au bloc soviétique. En dépit de ces efforts, la guerre contre un ennemi moins industrialisé a duré trois années. Gabriel Kolko nous a écrit que « [1]es Américains n'avaient pas prévu que leurs ennemis pourraient s'adapter, avec une économie de moyens remarquable, à leur technologie avancée, qu'en fait ils parviendraient presque à égaler leur puissance, infirmant ainsi toutes leurs expectatives quant à la longueur et aux coûts du conflit. $»{ }^{16}$ Ceci représentait un changement énorme dans la perspective américaine, puisqu'ils ne pouvaient pas facilement vaincre un ennemi avec la force économique comme ils l'ont fait auparavant. Le dernier changement psychologique lié à la guerre de Corée a été peutêtre le plus important et a été relevé tout au long de la Guerre froide. En fait, il était un thème commun pour chacun des blocs: l'absence d'une résolution du conflit. En Corée, les négociations de paix ont terminé en juillet 1953 avec un armistice après des centaines de sessions, trois années de blocage politique et la mort du chef soviétique. Cependant, la Corée est restée dans l'état ante bellum, divisée au trente-huitième parallèle, et il ne paraissait pas qu'aucun côté a émergé avec une victoire. Les Nations unies ont réussi à établir un arrêt d'hostilités, mais son ennemi n'était pas défait en aucun sens. La présence communiste dans la région n'a pas diminué, ni était-elle prête à négocier un retrait. Même si le président Truman a annoncé que «[t] here will be no profit for any people who follow the Communist dictatorship down its dark and bloody path ${ }^{17}$, le bloc occidental n'a pas pu tirer du profit de ce conflit non plus. L'impasse politique a été résout avec un document signé, mais le rideau de fer est resté intact. Dans ces circonstances, les États-Unis n'ont pas été capables de tourner la page de l'Histoire et commencer un nouveau chapitre dans ses relations avec la Chine et l'Union soviétique après la guerre de Corée. Au contraire, le résultat nul de la guerre a augmenté la tension entre les blocs et a créé un précédent pour la Guerre froide, puisque cette impasse politique était une situation qui s'est répétée plusieurs fois. Cet élément a marqué la politique américaine profondément puisque les États-Unis n'ont pas pu sentir la gloire du vainqueur ni le désespoir du vaincu; il y avait seulement la transition d'un affrontement à un autre et l'évolution des relations politiques dans un contexte de dissension.

Ici au Canada, on donne parfois à la guerre de Corée le surnom de « la guerre oubliée ». Puisque cette guerre n'a pas eu de « vainqueur » proprement dit, on néglige le rôle qu'elle a joué dans le contexte de la Guerre froide. Mais en réalité, cette guerre a contribué à celle-ci en modifiant la condition politique et psychologique des États-Unis. L'expérience de la guerre de Corée a aidé

\footnotetext{
${ }^{15}$ Harry S. Truman, “Our Aims and Our Hopes," Vital Speeches of the Day 16, no. 3 (1950): 711.

${ }^{16}$ Gabriel Kolko, Un siècle de guerres : Politique, conflit et société depuis 1914 (Saint-Nicolas, QC : Les presses de l’Université Laval, 1994), 356-57.

${ }^{17}$ Harry S. Truman, “Our Aims and Our Hopes," Vital Speeches of the Day 16, no. 3 (1950): 712.
} 
les États-Unis à se placer plus précisément sur l'échiquier politique, à mieux comprendre les stratégies de leurs ennemis, à évaluer le pouvoir des Nations unies, à réévaluer leurs relations avec les chefs politiques, et à se débarrasser de quelques illusions sur la façon de faire la guerre. Les Américains n'ont pas connu la victoire, mais ils sont sortis de cette guerre plus préparés pour les décennies difficiles qui l'ont suivi. J'espère qu'ils ne l'oublieront jamais.

\section{Bibliographie}

\section{1) Ouvrages :}

Fontaine, André. Histoire de la guerre froide : De la guerre de Corée à la crise des alliances 1950-1963. Paris : Librairie Arthème Fayard, 1967.

Fontaine, André. La guerre froide : 1917-1991. Paris : Éditions de La Martinière, 2006.

Kolko, Gabriel. Un siècle de guerres : Politique, conflits et société depuis 1914. Trans. Johanne Patry. Saint-Nicolas, QC : Les Presses de l’Université Laval, 1994.

\section{2) Articles :}

Fautua, David T. « The 'Long Pull' Army : NSC-68, the Korean War, and the Creation of the Cold War U.S. Army. » The Journal of Military History 61, no. 1 (1997) : 93120 (récupéré avec JSTOR)

Ovodenko, Alexander. « (Mis)interpreting Threats : A Case Study of the Korean War. » Security Studies 16, no.2 (2007) : 254-286 (récupéré avec Academic Search Complete)

\section{3) Autres sources :}

Truman, Harry S. « Our Aims and Our Hopes. » Vital Speeches of the Day 16, no. 3 (1950) : 709-712 (récupéré avec Academic Search Complete) 